\title{
The impact of a delay in intensive care unit admission for community-acquired
} pneumonia

\author{
J. Phua, W.J. Ngerng and T.K. Lim
}

ABSTRACT: The primary objective of the present study was to evaluate the effect on hospital mortality of a delay in intensive care unit (ICU) admission for severe community-acquired pneumonia (CAP). The secondary objectives were to assess if such delays were associated with treatment variations by the emergency department (ED) and deterioration in the general wards, and to evaluate the prognostic ability of the Infectious Disease Society of America (IDSA)/ American Thoracic Society (ATS) minor criteria.

We retrospectively compared patients who were admitted straight from the ED to the ICU (direct group, $n=54$ ) and those who were first admitted from the ED to the general wards before ICU transfer (delayed group, $n=49$ ), over 2.5 yrs.

Even after excluding patients who required mechanical ventilation and/or vasopressors at the ED, delayed ICU admission was an independent predictor of hospital mortality (OR 9.61). The delayed group received fewer fluid boluses in the ED and rapidly deteriorated in the general wards. The presence of $\geqslant 3$ IDSA/ATS minor criteria was associated with increased mortality in the delayed group.

In conclusion, prompt recognition of severe CAP using the IDSA/ATS minor criteria, followed by aggressive management at the ED and direct ICU admission, are all crucial toward improving outcomes.

KEYWORDS: Admission, emergency treatment, intensive care unit, mortality, pneumonia, resuscitation

pproximately $10 \%$ of hospitalised patients with community-acquired pneumonia (CAP) are admitted to the intensive care unit (ICU) [1-5]. The mortality for this group of patients is extremely high and ranges $20-50 \%$ in some studies [6]. Although the Infectious Disease Society of America (IDSA) and the American Thoracic Society (ATS) issued guidelines in 2007 which recommended that patients with severe CAP be promptly admitted to the ICU [1], onethird to a half of such patients are in fact first transferred from the emergency department (ED) to the general wards $[7,8]$. Part of the problem is that while patients who require emergent mechanical ventilation and/or vasopressors (defined by the IDSA/ATS as major criteria for severe CAP) and hence direct ICU admission may easily be identified [2], clinical findings at the ED which suggest a rapidly progressive pneumonia are often more subtle. Accordingly, numerous clinical criteria for severe CAP requiring ICU admission have recently been proposed $[3,9,10]$, including the IDSA/ATS minor criteria $[1,11,12]$.

Ironically, data on the impact on outcomes of the timing of ICU admission for severe CAP remain sparse. Two recent studies demonstrated that delayed ICU transfer was indeed associated with increased mortality [7, 13]. Nevertheless, ICU admission policies and patient epidemiology vary widely across the world [1]. Moreover, while one could attribute the difference in mortality between direct and delayed ICU admission to early management in the ICU, do other factors pertaining to the ED and the general wards matter as well, and can clinical scores be used to triage such patients more accurately?

We therefore conducted a study of patients who were admitted to our ICU for CAP. Our primary

\section{AFFILIATIONS}

Division of Respiratory and Critical Care Medicine, Dept of Medicine, National University Hospital, National University Health System, Singapore.

\section{CORRESPONDENCE}

T.K. Lim

Division of Respiratory and Critical Care Medicine

Dept of Medicine

National University Hospital National University Health System

5 Lower Kent Ridge Road

Singapore 119074

E-mail: tow_keang_lim@

nuhs.edu.sg

Received:

Sept 292009

Accepted after revision:

Feb 122010

First published online:

Feb 252010 
objective was to evaluate the effect of a delay in ICU admission on hospital mortality. Our secondary objectives were, first, to assess if such a delay was associated with variations in treatment by the ED and deterioration in the general wards which might contribute to poorer outcomes, and, secondly, to evaluate the prognostic ability of the IDSA/ATS minor criteria in these patients.

\section{PATIENTS AND METHODS}

\section{Study population}

We retrospectively reviewed all patients with CAP who were admitted to the 12-bed medical ICU of our university hospital (National University Hospital, National University Health System, Singapore) July 2005-December 2007. We classified the patients into the direct group (admitted straight from the ED to the ICU) and the delayed group (first admitted from the ED to the general wards before ICU transfer). To eliminate confounding by nosocomial complications, we excluded patients who were admitted to the ICU $>72 \mathrm{~h}$ after presentation at the ED. Some patients were included in a separate study conducted 2004-2007; this previous study, however, included non-critically ill patients and excluded those who were intubated or started on vasopressors in the ED [11]. The present study was approved by our institutional review board.

We defined CAP as an acute infection of the pulmonary parenchyma, presenting with an acute infiltrate on the chest radiograph and at least two of the following: fever $\left(\geqslant 38^{\circ} \mathrm{C}\right)$, hypothermia $\left(<36^{\circ} \mathrm{C}\right)$, rigours, sweats, new cough or change in colour of respiratory secretions, chest discomfort, or dyspnoea. We excluded patients who were hospitalised $<14$ days before the onset of symptoms, immunocompromised patients (with HIV infection or haematological malignancies, undergoing chemotherapy, or on steroids equivalent to $\geqslant 10 \mathrm{mg}$ per day of prednisolone for a month) and patients with tuberculosis.

\section{Clinical management}

Patient management at the ED was left to the discretion of the emergency medicine physicians. Protocols akin to the Surviving Sepsis Campaign's resuscitation bundles did not exist $[14,15]$. In general, blood cultures were performed and empiric antibiotics, which usually included a $\beta$-lactam antibiotic, e.g. a third generation cephalosporin, were administered within the ED. While an initial bolus of $20 \mathrm{~mL} \cdot \mathrm{kg}^{-1}$ body weight of crystalloids was recommended for shock, early

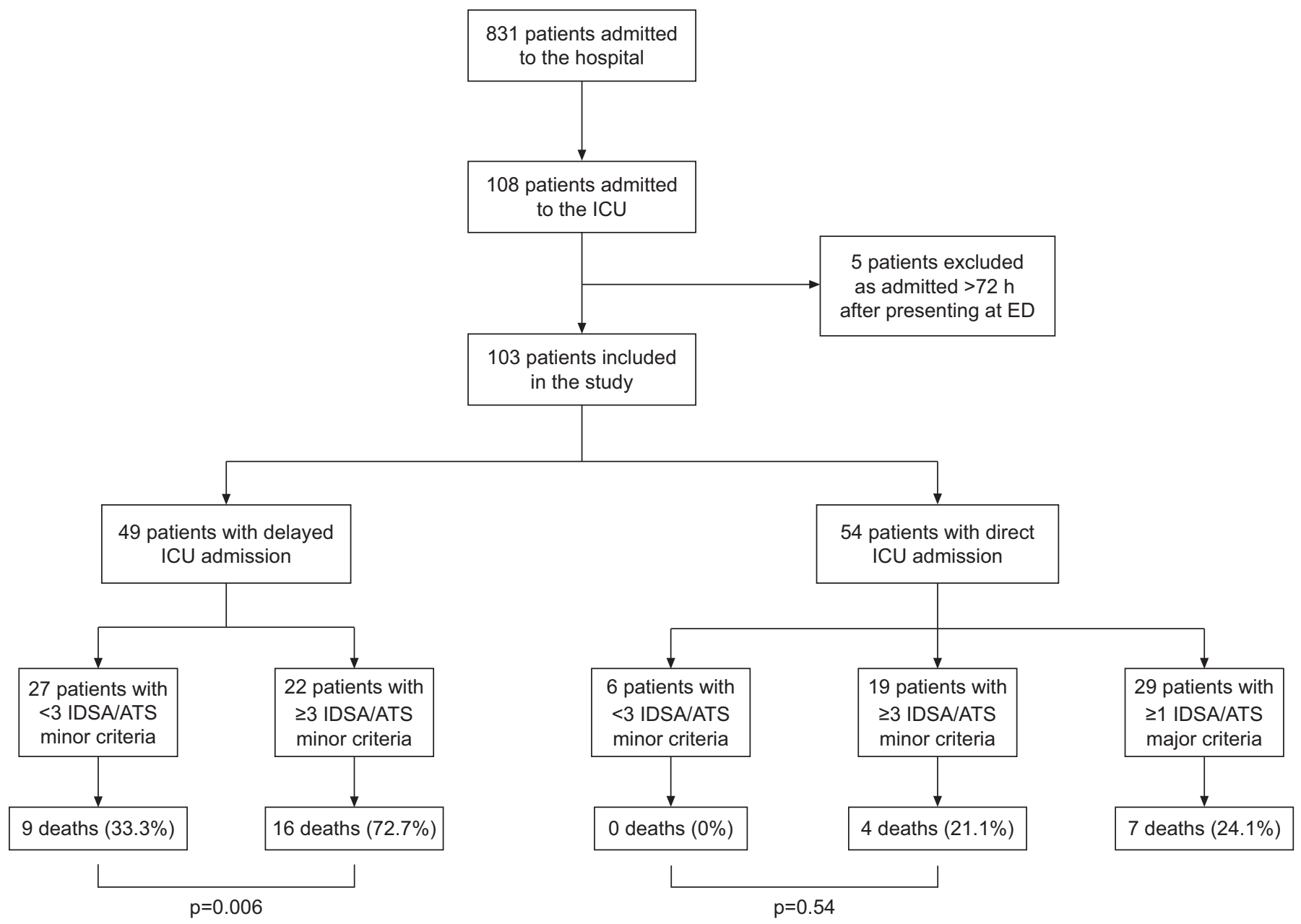

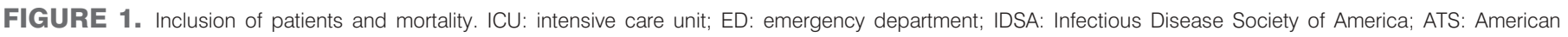
Thoracic Society. 


\begin{tabular}{|c|c|c|c|c|c|}
\hline Patients & 49 & 54 & & 25 & \\
\hline \multicolumn{6}{|l|}{ Demographics } \\
\hline Female sex & $13(26.5)$ & $14(25.9)$ & 0.94 & $6(24.0)$ & 0.81 \\
\hline Nursing home resident & $0(0)$ & $3(5.6)$ & 0.24 & $0(0)$ & NA \\
\hline \multicolumn{6}{|l|}{ Comorbid illnesses } \\
\hline Heart failure & $14(28.6)$ & $9(16.7)$ & 0.15 & $4(16.0)$ & 0.23 \\
\hline Cerebrovascular disease & $8(16.3)$ & $5(9.3)$ & 0.28 & $2(8.0)$ & 0.48 \\
\hline Neoplastic disease & $2(4.1)$ & $5(9.3)$ & 0.44 & $1(4.0)$ & 1.00 \\
\hline Heart rate beats $\cdot \mathrm{min}^{-1}$ & $106.9 \pm 21.0$ & $115.3 \pm 24.7$ & 0.07 & $115.9 \pm 24.0$ & 0.10 \\
\hline Respiratory rate breaths. $\min ^{-1}$ & $22.5 \pm 4.8$ & $25.4 \pm 6.7$ & 0.01 & $24.8 \pm 4.5$ & 0.04 \\
\hline Systolic blood pressure $\mathrm{mmHg}$ & $124.5 \pm 33.2$ & $106.5 \pm 35.5$ & 0.009 & $106.3 \pm 37.2$ & 0.04 \\
\hline Diastolic blood pressure $\mathrm{mmHg}$ & $63.9 \pm 15.8$ & $60.3 \pm 19.8$ & 0.31 & $62.0 \pm 20.7$ & 0.66 \\
\hline Temperature ${ }^{\circ} \mathrm{C}$ & $37.7 \pm 1.1$ & $37.5 \pm 1.4$ & 0.34 & $37.6 \pm 1.4$ & 0.72 \\
\hline $\mathrm{Sp}, \mathrm{O}_{2} / \mathrm{Fl}_{1} \mathrm{O}_{2}$ ratio $\%$ & $299.2 \pm 140.4$ & $169.7 \pm 123.4$ & $<0.001$ & $210.5 \pm 139.5$ & 0.01 \\
\hline \multicolumn{6}{|l|}{ Laboratory and radiological findings at the ED } \\
\hline Blood urea nitrogen $\mathrm{mmol} \cdot \mathrm{L}^{-1}$ & $12.7 \pm 12.9$ & $12.7 \pm 9.0$ & 0.99 & $13.5 \pm 9.1$ & 0.78 \\
\hline Sodium mmol. $\mathrm{L}^{-1}$ & $133.2 \pm 5.6$ & $132.9 \pm 6.1$ & 0.77 & $130.7 \pm 5.8$ & 0.08 \\
\hline Glucose $\mathrm{mmol} \cdot \mathrm{L}^{-1}$ & $10.1 \pm 6.7$ & $11.0 \pm 13.1$ & 0.69 & $11.2 \pm 11.8$ & 0.63 \\
\hline Haematocrit \% & $38.0 \pm 6.5$ & $38.0 \pm 6.2$ & 0.98 & $37.1 \pm 6.6$ & 0.55 \\
\hline
\end{tabular}

goal-directed therapy was not routinely practised [16]. The only definite indications for ICU admission were the need for mechanical ventilation and vasopressors. Otherwise, referral to the ICU depended on physician judgement.

Patients admitted to the general wards were managed by respiratory physicians during the day and the general medicine team on call after office hours. There was one staff nurse and one assistant nurse to 12 patients. Parameters were monitored at $1-6 \mathrm{~h}$ intervals. A macrolide was usually added to the previously administered $\beta$-lactam antibiotic if not already prescribed. Patients who remained unstable or deteriorated were transferred to the ICU.

This was a closed ICU with a 1:2 nurse-to-patient ratio and managed $24 \mathrm{~h}$ by accredited intensivists. Patients were treated according to the Surviving Sepsis Campaign guidelines [14, 15], and ventilated using a lung-protective strategy [17]. Empiric antibiotics were often escalated at the ICU to one of the following combinations: carbapenem/respiratory fluoroquinolone, carbapenem/macrolide, ceftazidime/respiratory fluoroquinolone, or ceftazidime/amoxicillin-clavulanate/macrolide.

\section{Data collection}

The following data were charted via medical record review into standardised forms: demographics (age, sex, nursing home residence), comorbid illnesses (heart failure, cerebrovascular, neoplastic, renal or liver diseases), the first available vital signs (mental status, heart rate, respiratory rate, blood pressure, temperature, ratio of arterial oxygen saturation measured by pulse oximetry $\left(\mathrm{Sp}_{\mathrm{p}} \mathrm{O}_{2}\right)$ to the fraction of inspired oxygen $\left(\mathrm{FI}, \mathrm{O}_{2}\right)$, laboratory findings (blood urea nitrogen, sodium, glucose, haematocrit, white blood cell and platelet count, arterial $\mathrm{pH}$ ) and radiological findings (pleural effusion, unilobar versus multilobar infiltrates) at the ED. 
TABLE 2 Treatment measures instituted

\begin{tabular}{|c|c|c|c|c|c|}
\hline Treatment measure & $\begin{array}{l}\text { Delayed ICU } \\
\text { admission, all } \\
\text { patients }\end{array}$ & $\begin{array}{c}\text { Direct ICU } \\
\text { admission, all } \\
\text { patients }\end{array}$ & p-value ${ }^{\#}$ & $\begin{array}{l}\text { Direct ICU admission, no } \\
\text { IDSA/ATS major criteria }\end{array}$ & p-value \\
\hline Patients & 49 & 54 & & 25 & \\
\hline Intubation at the ED & $0(0)$ & $21(38.9)$ & $<0.001$ & $0(0)$ & NA \\
\hline Vasopressors started at the ED & $0(0)$ & $17(31.5)$ & $<0.001$ & $0(0)$ & NA \\
\hline Appropriate antibiotics given on day $1^{\S}$ & $45(91.8)$ & $50(92.6)$ & 1.00 & $25(100.0)$ & 0.29 \\
\hline
\end{tabular}

We documented the Pneumonia Severity Index (PSI) [18], and classified severe CAP as those with one or more out of the two IDSA/ATS major criteria (need for mechanical ventilation or vasopressors) or three or more out of the minor criteria (confusion, uraemia, tachypnoea, hypotension, hypoxaemia, multilobar infiltrates, leukopenia, thrombocytopenia and hypothermia) at the ED [1].

We recorded the following treatment measures at the ED: intubation, vasopressors, fluid boluses and antibiotics. We also recorded all antibiotics administered on day 1 of presentation and microbiology results. We defined the antibiotic therapy as inappropriate if organisms were cultured that were not susceptible to the antibiotics.

We charted the time spent at the ED for all patients, and the time spent in the general wards before ICU transfer for the delayed group. As a measure of the pace of deterioration, for delayed patients with less than three IDSA/ATS minor criteria at the ED, we recorded the lag time from arrival at the general wards to the fulfilment of three or more minor criteria. For delayed patients who already met three or more minor criteria at the ED, we recorded the corresponding lag time to the accumulation of an additional minor criterion. We charted the final reasons for ICU transfer, including the number of major and minor criteria at the time of transfer.

The outcome measures were all-cause hospital mortality, length of stay in the hospital and ICU, duration of mechanical ventilation, need for mechanical ventilation and vasopressors at any time during the hospital stay, and the Acute Physiology and Chronic Health Evaluation (APACHE) II score for the first $24 \mathrm{~h}$ in the ICU.

\section{Data analyses}

We have expressed variables as $\mathrm{n}(\%)$, mean $\pm \mathrm{SD}$ and median (interquartile range (IQR)). We performed two-sided univariate analyses using the Chi-squared test, Fisher's exact test, t-test, paired t-test, Mann-Whitney U-test and Wilcoxon signed-rank test where appropriate. Comparisons via univariate analyses were first made between the delayed and direct groups in the following: baseline characteristics, treatment measures and patient outcomes. As we were interested in the patients who did not require mechanical ventilation or vasopressors at the ED, we repeated the comparisons between the delayed and direct groups after excluding patients with any IDSA/ATS major criteria. We constructed Kaplan-Meier curves for survival in the two groups and derived the hazard ratio using Cox regression. We then performed univariate analyses among these patients on the following variables to compare survivors versus nonsurvivors: demographics, comorbid illnesses, vital signs, laboratory findings, radiological findings at the ED, PSI, whether or not there were three or more IDSA/ATS minor criteria at the ED, treatment measures including the use of macrolides, and importantly, whether the patients were in the delayed or direct groups. To identify the independent predictors of hospital mortality, we entered variables with $\mathrm{p}$-values $<0.10$ into a forward logistic regression analysis using an entry level of 0.05 and a removal level of 0.10 . A p-value of $<0.05$ was considered significant. We used SPSS version 15.0 (SPSS Inc., Chicago, IL, USA).

\section{RESULTS}

As detailed in figure 1, 103 patients were included in the study: 54 in the direct group and 49 in the delayed group. The 29 patients who fulfilled one or more IDSA/ATS major criteria at the ED were restricted to the direct group, leaving 74 patients without any major criteria.

Overall, patients in the delayed group were older, less likely to have unstable vital signs, less acidaemic, and had numerically fewer PSI points (table 1). After excluding the patients with one or more IDSA/ATS major criteria, as far as the minor criteria were concerned, patients in the delayed group had less severe tachypnoea, hypotension and hypoxaemia.

Table 2 compares the treatment measures instituted in the delayed and the direct groups. The delayed group was given fewer fluid boluses and started on broad spectrum antibiotics less frequently in the ED than the direct group. The antibiotics given at the ED and microbiology data are shown in table a of the electronic supplementary data. When all antimicrobials administered on day 1 of presentation were considered, there was no difference in the frequency of macrolide use or inappropriate antibiotic administration between both groups. 


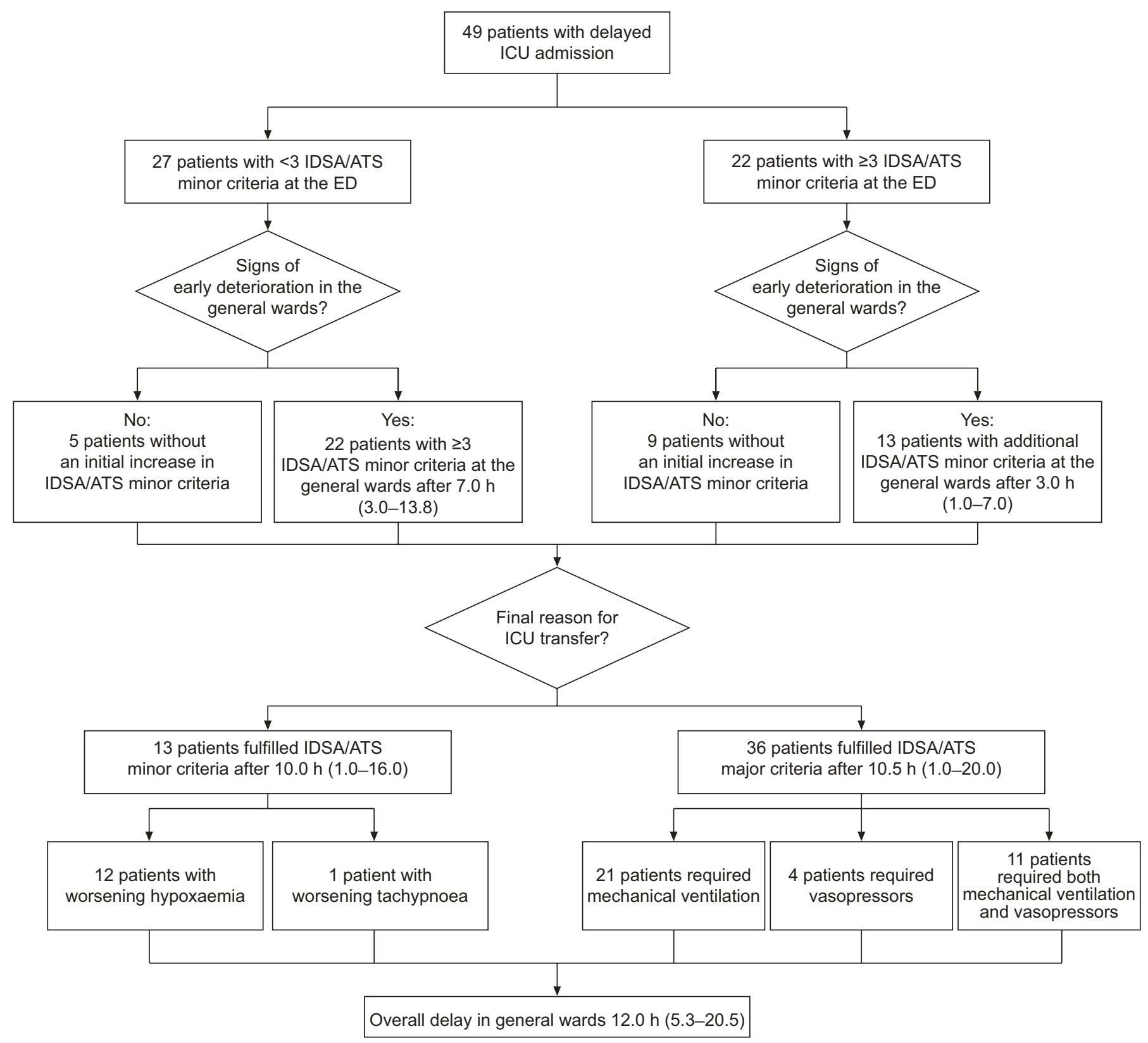

FIGURE 2. Clinical deterioration in the delayed group in the general wards. Data are presented as median (interquartile range). ICU: intensive care unit; IDSA: Infectious Disease Society of America; ATS: American Thoracic Society; ED: emergency department.

The delayed and direct groups spent a median of $3.5 \mathrm{~h}$ (IQR 3.06.0) and $3.5 \mathrm{~h}$ (IQR 2.2-5.0) in the $\mathrm{ED}$, respectively $(\mathrm{p}=0.11)$. After transfer to the general wards, the median number of IDSA/ATS minor criteria fulfilled by the delayed group increased from 2 (IQR 2-3) at the ED to 4 (IQR 3-5) at the time of ICU transfer $(p<0.001)$. More than half of these patients fulfilled further minor criteria within only a short time, several hours before ICU transfer (fig. 2). The final reasons for ICU transfer after a period of progressive deterioration were the fulfillment of additional minor criteria in 13 patients and one or more major criteria in 36 patients. Overall, the delayed group spent a median of $12.0 \mathrm{~h}$ (IQR 5.3-20.5) in the general wards.

Hospital mortality was higher in the delayed group than the direct group (51.0 versus 20.4\%; p=0.001) (table 3).
Kaplan-Meier survival curves for patients presenting without any IDSA / ATS major criteria show an early mortality difference between the two groups, which became progressively greater over time (fig. 3). Among these patients, the delayed group required mechanical ventilation more frequently and for more days than the direct group (table 3). The APACHE II score on day 1 of ICU admission was numerically, though not statistically, significantly higher in the delayed group.

Table 4 shows the predictors of mortality on univariate and multivariate analyses of the 74 patients without any IDSA/ ATS major criteria at the ED. The final independent predictors of hospital mortality in the logistic regression model were the PSI at the ED and the presence of a delayed ICU admission (OR 9.61, 95\% CI 2.32-39.78; $\mathrm{p}=0.002$ ). 
TABLE 3 Patient outcomes

\begin{tabular}{|c|c|c|c|c|c|}
\hline Outcome & $\begin{array}{l}\text { Delayed ICU } \\
\text { admission, } \\
\text { all patients }\end{array}$ & $\begin{array}{l}\text { Direct ICU } \\
\text { admission, } \\
\text { all patients }\end{array}$ & p-value ${ }^{\#}$ & $\begin{array}{c}\text { Direct ICU } \\
\text { admission, no IDSA/ } \\
\text { ATS major criteria }\end{array}$ & p-value \\
\hline Patients & 49 & 54 & & 25 & \\
\hline Hospital mortality & $25(51.0)$ & $11(20.4)$ & 0.001 & $4(16.0)$ & 0.004 \\
\hline Hospital length of stay days & $12.0(6.5-19.0)$ & $9.5(6.0-18.5)$ & 0.49 & $10.0(5.0-27.0)$ & 0.69 \\
\hline Need for mechanical ventilation in the ICU & $44(89.8)$ & $42(77.8)$ & 0.10 & $17(68.0)$ & 0.03 \\
\hline Need for vasopressors in the ICU & $35(71.4)$ & $40(74.1)$ & 0.76 & $15(60.0)$ & 0.32 \\
\hline APACHE II score on day 1 in the ICU & $26.3 \pm 8.0$ & $24.0 \pm 8.5$ & 0.16 & $23.4 \pm 9.7$ & 0.18 \\
\hline
\end{tabular}

Data are presented as $n, n(\%)$, median (interquartile range) or mean \pm SD, unless otherwise stated. ICU: intensive care unit; IDSA: Infectious Disease Society of America; ATS: American Thoracic Society; APACHE: Acute Physiology and Chronic Health Evaluation. *: refers to comparison between all patients in the delayed group and the direct group; ": refers to comparison between all patients in the delayed group and only patients presenting without IDSA/ATS major criteria in the direct group.

The association between the presence of three or more IDSA/ ATS minor criteria and hospital mortality did not achieve statistical significance in either univariate $(p=0.06)$ or multivariate $(\mathrm{p}=0.09)$ analyses of these 74 patients (table 4 ). Notwithstanding, in the delayed group, mortality was $72.7 \%$ in patients with three or more minor criteria, which was significantly higher than the $33.3 \%$ mortality in those with less than three minor criteria $(p=0.006)$ (fig. 1$)$. In the direct group, the mortality was $21.1 \%$ in patients with three or more minor criteria and $0 \%$ in those with less than three minor criteria $(\mathrm{p}=0.54)$.

\section{DISCUSSION}

In the present study, almost half of the critically ill patients with CAP suffered a delay in ICU admission. Even after excluding patients who required emergent mechanical ventilation and/or vasopressors at the ED, this delay was an independent predictor of hospital mortality (OR 9.61). The delayed group received less intensive treatment at the ED and rapidly deteriorated in the general wards. The presence of

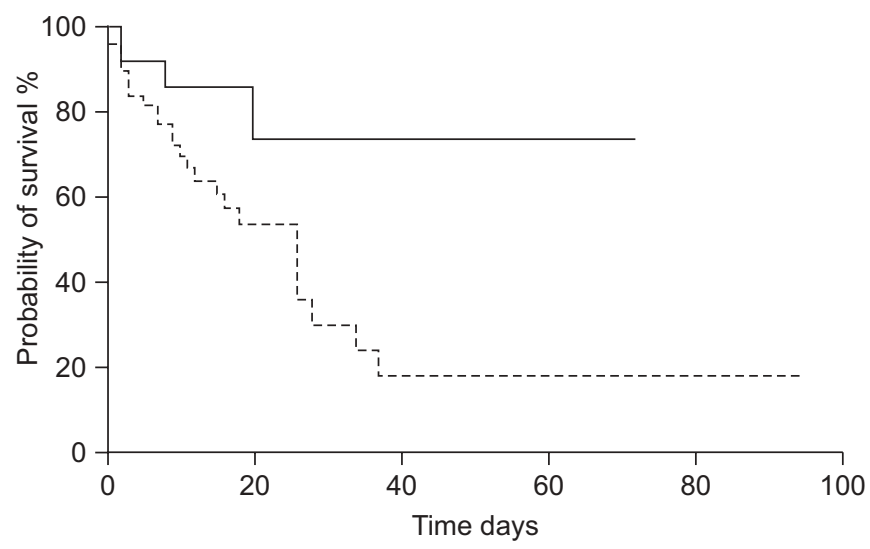

FIGURE 3. Kaplan-Meier curves for survival from the time of hospital admission for the 74 patients presenting without Infectious Disease Society of America/American Thoracic Society major criteria. —: direct intensive care unit (ICU) admission; ---: delayed ICU admission. Hazard ratio 3.39 (95\% Cl 1.17-9.78). three or more IDSA/ATS minor criteria at the ED was associated with increased mortality in the delayed group.

Although the 2007 IDSA/ATS guidelines recommend that patients with severe CAP be promptly admitted to the ICU [1], data on the dangers of delayed ICU admission are sparse. Two recent studies by RENAUD et al. [7] and RESTREPO et al. [13] found that such delays were associated with increased mortality and a longer length of stay. The results of the present study are consistent the data of these studies. In our logistic regression model, aside from delayed ICU admission, the other predictor of mortality was the PSI, a robust prognostic score [18], which adds strength to our findings. The question that remains unanswered, however, is why direct ICU admission should lead to improved outcomes.

While due credit may be given to early care in the ICU $[19,20]$, the present study explores, for the first time, two other reasons for the improved outcomes associated with direct ICU admission.

First, management in the ED was more aggressive for the direct group than the delayed group. The former received more volume resuscitation, a key life-saving measure in severe sepsis [16]. The amount of fluids administered was inversely associated with mortality on univariate analysis. Though there were fewer hypotensive patients in the delayed group than in the direct group, blood pressure does not reflect tissue perfusion well [21], and lactate levels were not systematically measured by the ED. In addition to more ventilatory and vasopressor support, antibiotics were more consistently administered in the ED for the direct than for the delayed group. Although antibiotics were not predictors of mortality in the present study, delay in antimicrobial therapy has been associated with decreased survival in severe sepsis [22]. The importance of aggressive management at the ED becomes even clearer when one considers the frequent need to board patients at the ED due to the widespread shortage of ICU beds [19, 23].

Secondly, many patients in the delayed group deteriorated in the general wards, as evidenced by the rapid accumulation of additional IDSA/ATS minor criteria, several hours before ICU 


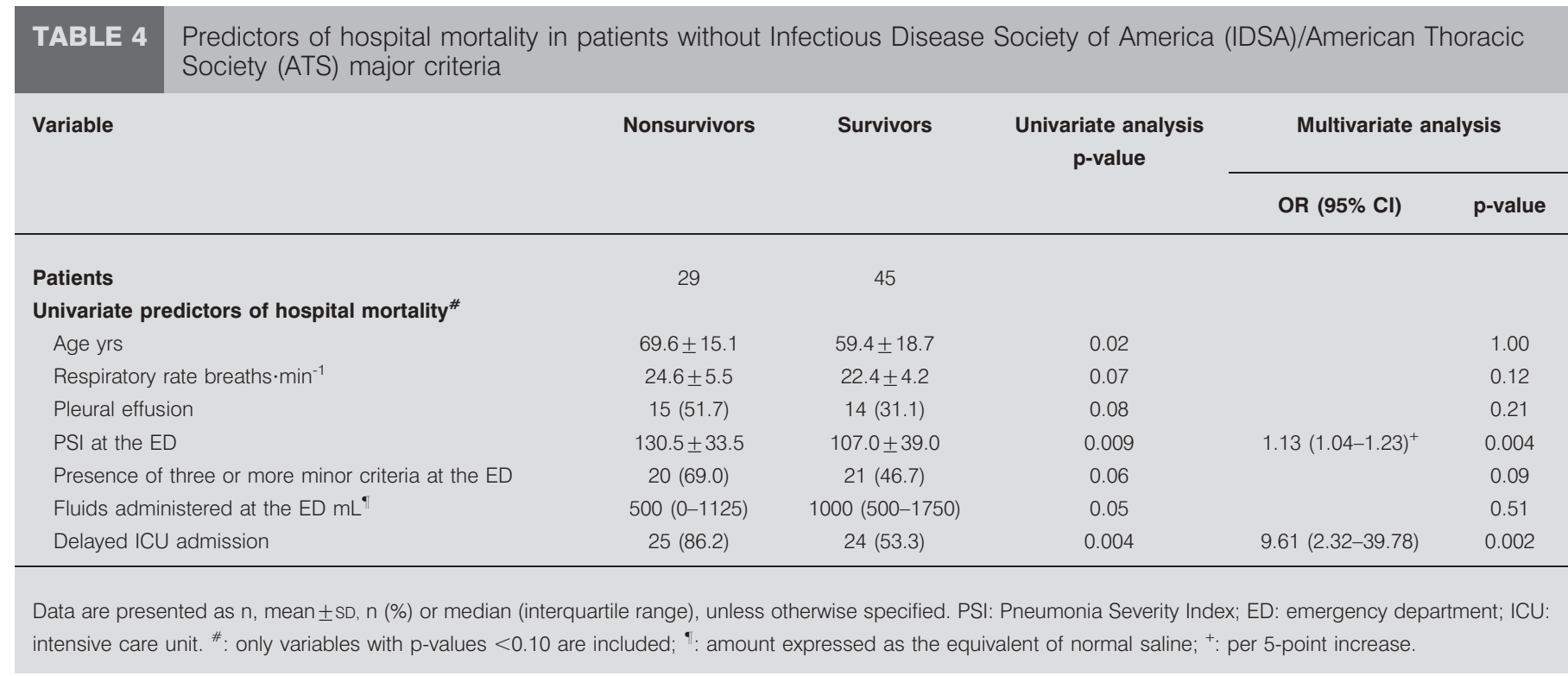

transfer. This delay may have contributed to the fact that 36 out of the 49 patients in the delayed group eventually required emergency mechanical ventilation and/or vasopressors at the time of ICU transfer. While the delayed group had lower PSI scores at the ED than the direct group, there was no longer any difference in the severity of illness by day one of the ICU stay, based on the APACHE II score. Indeed, as illustrated by the survival curves, the mortality difference between the two groups progressively increased even after 1 month, suggesting that inadequate initial treatment could have contributed to subsequent complications, including organ failures and superinfections. These findings are not surprising, given the relative lack of intensive monitoring and treatment in the general wards. It has previously been shown that patients who developed septic shock in the general wards fared poorer than those in the ICU [24].

Since delayed ICU admission is an independent predictor of hospital mortality, early recognition of severe CAP is crucial, yet signs of severity are often subtle in patients without frank respiratory failure or shock. Interestingly, although none of the six patients with less than three IDSA/ATS minor criteria, who were directly admitted to the ICU, died, there was no significant difference in mortality between these patients and those with three or more minor criteria in the direct group. However, in the delayed group, mortality was significantly higher in patients with three or more than those with less than three minor criteria. Taken together, these findings mandate a low threshold for direct ICU admission for patients with three or more minor criteria.

The limitations of the present study must, however, be acknowledged. First, because it focused only on ICU patients, it did not evaluate the outcomes of patients with three or more IDSA/ATS minor criteria who might have stabilised or died without ICU admission. Two studies that enrolled both ICU and non-ICU patients, and which recently validated the minor criteria, are relevant in this setting [11, 12]. The first study, also by our group, found that $45.2 \%$ of all comers to the ED who did not require emergent intubation or vasopressors, and who fulfilled three or more minor criteria, eventually died [11]. This high mortality rate, in our opinion, supports our call for direct ICU admission for such patients. Secondly, although the present study is the second-largest to date on patients with delayed ICU admission for CAP, it is retrospective, from a single centre, and has a relatively small sample size. To circumvent this limitation, we made every attempt to ensure accurate data collection using standardised forms. Thirdly, we did not compare the treatment provided in the ICU between the direct and delayed groups. Nevertheless, as our ICU uses protocols which follow the Surviving Sepsis Campaign guidelines closely [14, 15], we are confident that the management should not differ across groups. Lastly, we did not compare other predictive indices for severe CAP, such as SMART-COP (systolic blood pressure, multilobar chest radiography involvement, albumin level, respiratory rate, tachycardia, confusion, oxygenation, arterial $\mathrm{pH}$ ), the severe CAP (SCAP) prediction rule and the PIRO (predisposition, insult, response, organ dysfunction) score $[3,4,9,10]$.

We shall conclude with a discussion of the clinical implications of the present study. The decision for ICU admission is relatively straightforward for patients with severe CAP who require mechanical ventilation and/or vasopressors at the ED. However, most patients do not fall into this category, but instead present with more subtle signs of severity. Our data clearly show that a delay in ICU admission for such patients independently predicts increased hospital mortality. This delay is associated with the provision of less intensive treatment at the ED and rapid deterioration in the general wards. Hence, in such patients, the IDSA/ATS minor criteria should be used to facilitate prompt recognition of severe CAP. The presence of three or more minor criteria should trigger aggressive management at the ED, including adequate fluid resuscitation, followed by direct ICU admission, all of which are crucial steps toward improving the outcomes of a frequently fatal disease.

\section{STATEMENT OF INTEREST}

None declared. 


\section{ACKNOWLEDGEMENTS}

The authors would like to express their appreciation to all healthcare workers involved in the care of patients with community-acquired pneumonia in the emergency department, the general wards and the medical intensive care unit of Singapore's National University Hospital.

\section{REFERENCES}

1 Mandell LA, Wunderink RG, Anzueto A, et al. Infectious Diseases Society of America/American Thoracic Society consensus guidelines on the management of community-acquired pneumonia in adults. Clin Infect Dis 2007; 44: Suppl. 2, S27-S72.

2 Liapikou A, Ferrer M, Polverino E, et al. Severe communityacquired pneumonia: validation of the Infectious Diseases Society of America/American Thoracic Society Guidelines to predict an intensive care unit admission. Clin Infect Dis 2009; 48: 377-385.

3 Charles PG, Wolfe R, Whitby M, et al. SMART-COP: a tool for predicting the need for intensive respiratory or vasopressor support in community-acquired pneumonia. Clin Infect Dis 2008; 47: 375-384

4 Yandiola PP, Capelastegui A, Quintana J, et al. Prospective comparison of severity scores for predicting clinically relevant outcomes for patients hospitalized with community-acquired pneumonia. Chest 2009; 135: 1572-1579.

5 Restrepo MI, Mortensen EM, Waterer GW, et al. Impact of macrolide therapy on mortality for patients with severe sepsis due to pneumonia. Eur Respir J 2009; 33: 153-159.

6 Rello J. Demographics, guidelines, and clinical experience in severe community-acquired pneumonia. Crit Care 2008; 12: Suppl. $6, \mathrm{~S} 2$.

7 Renaud B, Santin A, Coma E, et al. Association between timing of intensive care unit admission and outcomes for emergency department patients with community-acquired pneumonia. Crit Care Med 2009; 37: 2867-2874.

8 Ewig S, de Roux A, Bauer T, et al. Validation of predictive rules and indices of severity for community acquired pneumonia. Thorax 2004; 59: 421-427.

9 Espana PP, Capelastegui A, Gorordo I, et al. Development and validation of a clinical prediction rule for severe communityacquired pneumonia. Am J Respir Crit Care Med 2006; 174: 1249-1256.

10 Rello J, Rodriguez A, Lisboa T, et al. PIRO score for communityacquired pneumonia: A new prediction rule for assessment of severity in intensive care unit patients with community-acquired pneumonia. Crit Care Med 2009; 37: 456-462.
11 Phua J, See KC, Chan YH, et al. Validation and clinical implications of the IDSA/ATS minor criteria for severe community-acquired pneumonia. Thorax 2009; 64: 598-603

12 Brown SM, Jones BE, Jephson AR, et al. Validation of the Infectious Disease Society of America/American Thoracic Society 2007 guidelines for severe community-acquired pneumonia. Crit Care Med 2009; 37: 3010-3016.

13 Restrepo MI, Mortensen EM, Rello J, et al. Late admission to the ICU in patients with community-acquired pneumonia is associated with higher mortality. Chest 2009; 137: 522-527.

14 Dellinger RP, Carlet JM, Masur $\mathrm{H}$, et al. Surviving Sepsis Campaign guidelines for management of severe sepsis and septic shock. Crit Care Med 2004; 32: 858-873.

15 Dellinger RP, Levy MM, Carlet JM, et al. Surviving Sepsis Campaign: international guidelines for management of severe sepsis and septic shock: 2008. Crit Care Med 2008; 36: 296-327.

16 Rivers E, Nguyen B, Havstad S, et al. Early goal-directed therapy in the treatment of severe sepsis and septic shock. $N$ Engl J Med 2001; 345: 1368-1377.

17 Ventilation with lower tidal volumes as compared with traditional tidal volumes for acute lung injury and the acute respiratory distress syndrome. The Acute Respiratory Distress Syndrome Network. N Engl J Med 2000; 342: 1301-1308.

18 Fine MJ, Auble TE, Yealy DM, et al. A prediction rule to identify low-risk patients with community-acquired pneumonia. $N$ Engl J Med 1997; 336: 243-250.

19 Chalfin DB, Trzeciak S, Likourezos A, et al. Impact of delayed transfer of critically ill patients from the emergency department to the intensive care unit. Crit Care Med 2007; 35: 1477-1483.

20 Engoren $M$. The effect of prompt physician visits on intensive care unit mortality and cost. Crit Care Med 2005; 33: 727-732.

21 Antonelli M, Levy M, Andrews PJ, et al. Hemodynamic monitoring in shock and implications for management. International Consensus Conference, Paris, France, 27-28 April 2006. Intensive Care Med 2007; 33: 575-590.

22 Kumar A, Roberts D, Wood KE, et al. Duration of hypotension before initiation of effective antimicrobial therapy is the critical determinant of survival in human septic shock. Crit Care Med 2006; 34: 1589-1596.

23 Halpern NA, Pastores SM. Critical care medicine in the United States 2000-2005: an analysis of bed numbers, occupancy rates, payer mix, and costs. Crit Care Med 2009; 38: 65-71.

24 Lundberg JS, Perl TM, Wiblin T, et al. Septic shock: an analysis of outcomes for patients with onset on hospital wards versus intensive care units. Crit Care Med 1998; 26: 1020-1024. 